\title{
Molecular classification of endometrial carcinoma, is it the new era of precision medicine?
}

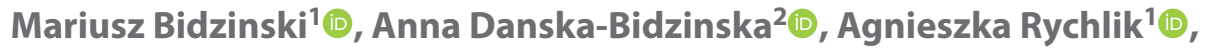 \\ Jolanta Kupryjanczyk ${ }^{3}$ (D), Michal Pyzlak ${ }^{3}$, Szymon Piatek ${ }^{1}$ \\ ${ }^{1}$ Department of Gynecologic Oncology, Maria Sklodowska-Curie National Research Institute of Oncology, Warsaw, Poland \\ ${ }^{2} 2^{\text {nd }}$ Chair and Department Obstetrics and Gynecology, Medical University of Warsaw, Poland \\ ${ }^{3}$ Pathology Department, Maria Sklodowska-Curie National Research Institute of Oncology, Warsaw, Poland
}

\begin{abstract}
For many years endometrial cancer has been subdivided into oestrogen — dependent (type I) and oestrogen — independent (type II), according to classical Bokhman classification. Histopathological evaluation including type and grade of tumour, along with clinical factors have been considered as very important prognostic factors that impact treatment decision. However, histologically similar tumours may have different outcomes. Recent molecular findings and new histopathological parameters have given new concept on risk stratification. The Cancer Genome Atlas Research Network (TCGA) of tumours have brought new insights into endometrial cancer management. Four molecular subgroups have been described: POLE ultramutated (POLE mut), p53 mutant (p53abn), mismatch repair deficient (MMRd) and non-specific molecular profile (NSMP). This new subdivision has been recently introduced in the European risk stratification system. Key words: molecular classif endometrial cancer; POLE mutations; p53 mutations; MMRd; NSMP
\end{abstract}

Ginekologia Polska 2022; 93, 2: 163-167

\section{INTRODUCTION}

Endometrial cancer $(\mathrm{EC})$ is the second most common gynaecologic malignancy that affects thousands of women globally. In 2018, 417367 new cases were diagnosed worldwide and 97370 patients died [1]. Although nearly $80 \%$ of EC patients are diagnosed in stage I-II according to 2018 FIGO classification [2], some of the apparently early-stage EC, have fatal outcomes. There are some several international guidelines concerning adjuvant treatment in early EC, however the recommendations are ambiguous. Guidelines of the European Society of Medical Oncology (ESMO) [3] or National Comprehensive Cancer Network (NCCN) [4] present a wide spectrum of options from patients' observations to adjuvant chemoradiotherapy.

Until now, the $4^{\text {th }}$ edition of the World Health Organization (WHO) classification of tumours of female reproductive organs was based on histological morphology completed by immunohistochemical prognostic markers [5]. The treatment indications were based on these findings, but in some cases, histopathological interobserver variations have been demonstrated. This fact explains the reason why scientists started to search for new prognostic factors to precise optimal indications to adjuvant therapy in EC patients. Recently, the Cancer Genome Atlas Research Network (TCGA) brought important knowledge regarding molecular profile of EC [6]. 373 cases of EC were analysed using next generations sequencing (NGS) test, and these cases were stratified to four different subgroups. These four subgroups include: ultra-mutated EC which presents pathogenic variants in the exonuclease domain of DNA polymerase-epsilon (POLE), hypermutated EC characterised by microsatellite instability (MSI), a low copy number with a low mutational burden and a high copy number with TP53 mutations. Validation of prognostic factors by molecular stratifications have restructured EC classifications. This project included a comprehensive analysis of endometrioid, serous and mixed histology. Nowadays, based on transcriptomic, genomic and proteomic characterization EC is categorised as follows: POLEmut EC (pathogenic polymerase - epsilon variants), MMRd EC (mismatch repair protein deficiency), NSMP EC 


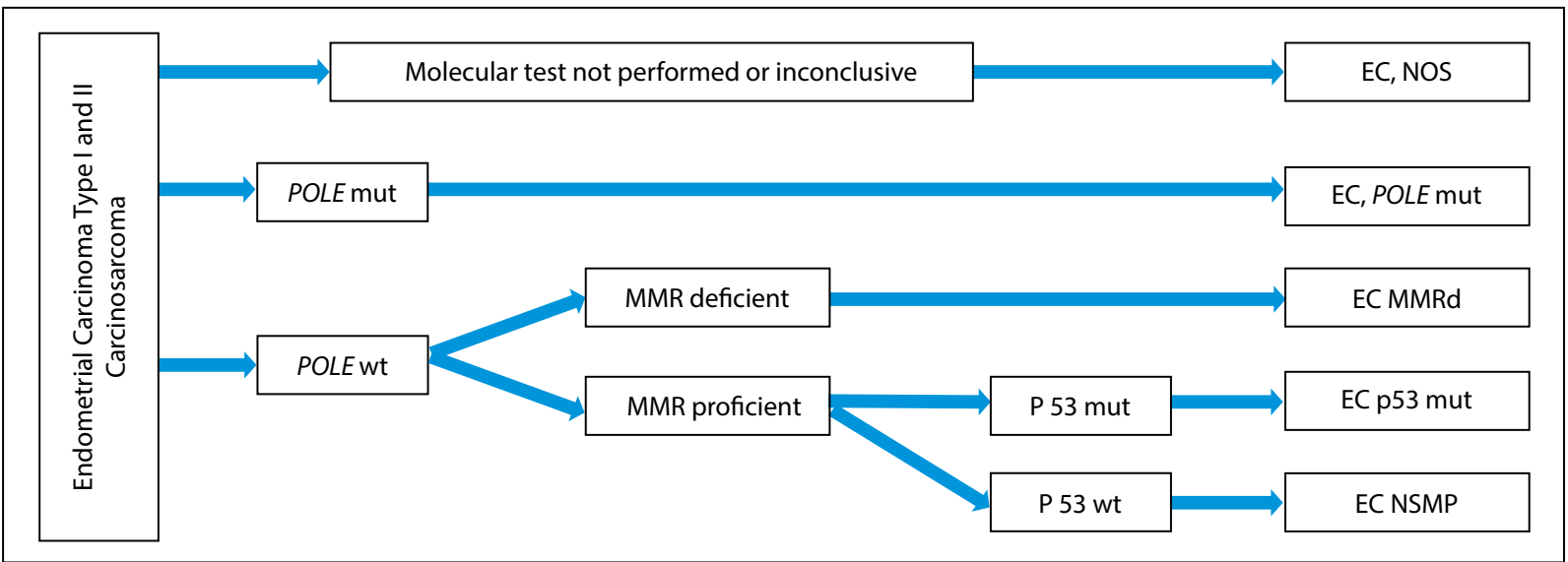

Figure 1. Proposition of diagnostic algorithm of endometrial cancer including molecular testing

(nonspecific molecular profile) and p53mut EC (mutation in p53). In case of inconclusive or not performed molecular test the term NOS (not otherwise specified) should be used. New algorithm of pathological/molecular examination of EC was presented on Figure 1.

\section{CLINICAL SIGNIFICANCE OF NEW MOLECULAR CLASSIFICATION OF ENDOMETRIAL CANCERS EC, POLE mut}

POLE mutated variants comprise about 7\% of all EC [6]. $P O L E$ gene encodes polymerase epsilon $(\varepsilon)$, which can correct DNA synthesis errors and helps protect against genome instability. Loss of function in the DNA polymerase $\varepsilon$ is important in tumorigenesis of EC. Tumours with POLE exonuclease domain mutations (EDMs) have shown to increase spontaneous mutation rates and are referred as "POLE ultra-mutated".

POLE mutations are assessed using PCR amplification and Sanger sequencing, while other subgroups of EC are evaluated by immunohistochemical staining [7]. Five hot spots were recognized: P286R, V411L, S297F, A456P and S459F. This variant of tumour despite the presence of poor pathologic features (high grade and deep myometrium invasion) has good prognosis with improved progression free survival (PFS) $[8,9]$. PORTEC 1 and 2 trials show that in intermediate and high intermediate groups of EC POLE mut and POLE wild type (wt) 10-years cancer specific survival were $97.7 \%$ and $89.7 \%(p=0.11)$, respectively [10]. POLE-mutant tumours have a risk of recurrence approximately one third of that in other types of EC. In PORTEC trials any of POLEmut grade 3 patient recurred in comparison with $30.9 \%$ of grade 3 tumours in the rest of subgroups. These findings support opinion that POLE mut EC has intrinsic factors beneficial for survival independent of adjuvant treatment [11]. In this type of EC high mutational load have been demonstrated and immunogenic reactions due to huge lymphocyte $T$ infiltration of tumour was observed [12]. Additionally, loss of increased radiation sensitivity in POLE mut embryonic stem cells was estimated [13]. Improved overall survival was also observed in POLE mut high risk EC [14]. Apart from mutations in POLE gene multiple alterations in molecular profile could be found in EC (POLEmut and p53 mut, POLEmut and MMRd, etc.) - known as double classifier. Those additional mutations do not influence survival; patients have good prognosis and should be managed as POLE mut [11].

\section{EC, MMRd}

This type of molecular profile is frequent in EC (approximately 25-30\%). It is defined by loss of nuclear expression of one or more MMR proteins (MLH1, MSH2, MSH6 and PMS2) within tumour cell $[6,15]$. MMRd EC has an intermediate prognosis. Similar to POLE mut also had abundance of tumour infiltrating lymphocytes (TILs). Exploratory analysis of NRG/GOG 210 study showed that adjuvant treatment improves PFS in MMRd EC in contrast to POLE mut [16]. Long term analysis of PORTEC 2 trial revealed similar effectiveness of vaginal brachyterapy in comparison with external beam radiotherapy (EBRT) in reducing pelvic lymph node recurrence risk in MMRd group in the absence of other unfavourable prognostic factors (substantial LVSI, p53 mut, L1CAM) [18]. Patients with high risk (HR) MMRd EC had no benefit from addition CT to EBRT [14]. Assessment of lympho-vascular space invasion (LVSI) in histopathologic protocol is crucial as it is one of the most important prognostic factors in EC. It should be stated that focal LVSI (single focus of LVSI around the tumour) have no impact on prognosis in opposite to substantial LVSI (extensive LVSI). Substantial LVSI is observed in up to $8.9 \%$ of MMRd EC [17]. In low-risk endometrial cancer patients, MMRd increases of ovarian metastasis and synchronous gonadal involvement [19]. 


\section{EC, NSMP}

Non-specific molecular profile (NSMP; also called low copy number) EC should be diagnosed in patients with p53 wild type expression, MMR proficient and absence of pathogenic mutations in POLE gene. This subtype has worse prognosis than the POLE mut and MMRd types. According to newly developed Proactive Molecular Risk Classifier for Endometrial Cancer (ProMisE), L1 cell-adhesion molecule (L1CAM) is significant indicator of high-risk disease in EC. In NSMP subgroup univariate analysis showed higher risk of fatal outcome in L1CAM positive patients compared to L1CAM negative counterpart. Disease specific survival in L1CAM positive group had HR $6.94(95 \% \mathrm{Cl}$ 2.56-18.74; $\mathrm{p}<0.001$ ) [18]. After hysterectomy, patients with p53 wt/NSMP, L1CAM-positive tumours were at similar risk for fatal outcome when compared to patients with $\mathrm{p} 53$ mut disease. These patients should be subjected to adjuvant therapy even if ESMO criteria indicate low risk group with no adjuvant treatment necessary. Adjuvant EBRT with or without chemotherapy depend on patients' status, should be used [20]. In cases of p53 wt/NSMP L1CAM negative, addition of chemotherapy did not improve survival [21].

\section{EC, p53 mut}

This high copy number type of EC presents a very aggressive course and the worst outcome. Comparing 5 years PFS between subgroups: p53 mut, POLE mut, MMRd and NSMP, the results were as follows: $50 \%, 98 \%, 74 \%$ and $76 \%$, respectively [21]. Budak et al. [22] showed that high p53 expression correlates with advanced stage of endometrial cancer. Adjuvant chemoradiotherapy (CRT) improved overall survival only in this subtype of EC. HR patients with $\mathrm{p} 53$ mut included to PORTEC 3 trial achieved better 5 years PFS in CRT group in comparison with radiotherapy group: $61 \%$ vs $37 \%$; $\mathrm{p}<0.001$ [14]. Additionally, amplification of ERBB2 gene is found quite often [21]. The prevalence of homologous recombination deficiency (HRD) had been determined in 46\% of EC p53 mut [23].

\section{NEW POTENTIAL TARGETED THERAPY IN EC}

Both MSI and POLE mut subtypes express essential immunogenicity because of a high mutational burden [24]. Ad- dition of PDL1 or anti PD-1 agents (atelizumab, nivolumab or pembrolizumab) can be effective in recurrent or metastatic MSI or POLE mutations EC [25-27].

In EC cases, showing the amplification of ERBB2 gene and overexpression of HER2, trastuzumab therapy may be used. Trastuzumab is a monoclonal antibody directed against the HER2 receptor. The ongoing study may confirm any benefit of adjusting trastuzumab therapy in recurrent EC. The trial including patients with stages III/IV or recurrent HER2 EC presents improved PFS from 8 to 13 months $(p=0.003)[27,28]$. Subgroup of EC $p 53$ mut serous type express germline $B R C A 1 / 2$ mutations, and therefore poly (ADP ribose) polymerase inhibitors may show any efficacy in treatment modality. Currently, several clinical trials investigating the efficacy of PARP inhibitors in recurrent or metastatic EC have been ongoing. Based on previous information from "ProMisE" (Proactive Molecular Risk Classifier for Endometrial Cancer), ESGO (European Society of Gynaecological Cancer), ESTRO (European Society for Radiotherapy and Oncology) and ESP (European Society of Pathology) prepared recommendations on risk stratification presented in Table 1.

The PORTEC 4a study aims to determine efficiency of molecular integrated risk profiles in endometrial cancer. This trial intends to compare molecular profile-based adjuvant therapy versus adjuvant treatment based on standard pathological characteristics.

\section{SUMMARY}

The new insights into molecular classification give novel information to better understand the biology of endometrial cancer. New stratifications based on molecular and clinical factors will allow to treat EC patients more precisely. New recommendations will hopefully help to avoid over an undertreatment of endometrial cancer patients. About $50 \%$ of patients in the POLE subgroup (excellent prognosis) were classified as ESMO high-risk, and about $25 \%$ of patients in the high copy number subgroup would be classified as ESMO low/intermediate risk. These data raise the question of proper adjuvant therapy. The EC molecular classification will permit to introduce a new therapeutic modality in these cases. Results of prospective PORTEC 4a trial are still awaited. 
Table 1. Risk stratification according to histopathological criteria and molecular classification [30] (by courtesy of International Journal of Gynaecological Cancer)

\begin{tabular}{|c|c|c|}
\hline Risk group & Molecular classification unknown & Molecular classification known* \\
\hline Low & $\begin{array}{l}\text { Stage IA endometrioid + low grade } \text { l }^{\&} \text { LVSI negative } \\
\text { or focal }\end{array}$ & $\begin{array}{l}\text { - Stage I-II POLEmut endometrial carcinoma, no residual } \\
\text { disease } \\
\text { - Stage IA MMRd/NSMP endometrioid carcinoma + } \\
\text { + low-grade }+ \text { LVSI negative or focal }\end{array}$ \\
\hline Intermediate & $\begin{array}{l}\text { Stage IB endometrioid + low-grade }{ }^{\&}+\text { LVSI negative } \\
\text { or focal } \\
\text { - Stage IA endometrioid + high-grade }{ }^{\&}+\text { LVSI negative } \\
\text { or focal } \\
\text { - Stage IA non-endometrioid (serous, clear cell, } \\
\text { undifferentiared carcinoma, cacinosarcoma, mixed) } \\
\text { without myometrial invasion }\end{array}$ & $\begin{array}{l}\text { Stage IB MMRd/NSMP endometrioid carcinoma + } \\
+ \text { low-grade\& + LVSI negative or focal } \\
\text { - Stage IA MMRd/NSMP endometrioid + high- } \\
\text { grade\& + LVSI negative or focal } \\
\text { - Stage IA p53abn and/or non-endometrioid (serous, } \\
\text { clear cell, undifferentiated carcinoma, carcinosarcoma, } \\
\text { mixed) without myometrial invasion }\end{array}$ \\
\hline High-intermediate & $\begin{array}{l}\text { - Stage I endometrioid + substantial LVSI regardless } \\
\text { of grade and depth of invasion } \\
\text { - Stage IB endometrioid high-grade }{ }^{\&} \text { regardless } \\
\text { of LVSI status } \\
\text { - Stage II }\end{array}$ & $\begin{array}{l}\text { Stage I MMRd/NSMP endometrioid } \\
\text { carcinoma + substantial LVSI regardless of grade } \\
\text { and depth of invasion } \\
\text { - Stage IB MMRd/NSMP endometrioid carcinoma } \\
\text { high-grade\& regardless of LVSI status } \\
\text { - Stage II MMRd/NSMP endometrioid carcinoma }\end{array}$ \\
\hline High & $\begin{array}{l}\text { - Stage III-IVA with no residual disease } \\
\text { Stage I-IVA non-endometrioid (serous, clear cell, } \\
\text { undifferentiared carcinoma, cacinosarcoma, mixed) } \\
\text { without myometrial invasion, and with no residual } \\
\text { disease }\end{array}$ & $\begin{array}{l}\text { - Stage III-IVA MMRd/NSMP endometrioid carcinoma } \\
\text { with no residual disease } \\
\text { - Stage I-IVA p53abn endometrial carcinoma with } \\
\text { myometrial invasion, with no residual disease } \\
\text { - Stage I-IVA NSMP/MMRd serous, undifferentiated } \\
\text { carcinoma, carcinosarcoma with myometrial invasion, } \\
\text { with no residual disease }\end{array}$ \\
\hline Advanced metastatic & $\begin{array}{l}\text { - Stage III-IVA with residual disease } \\
\text { - Stage IVB }\end{array}$ & $\begin{array}{l}\text { - Stage III-IVA with residual disease of any molecular type } \\
\text { - Stage IVB of any molecular type }\end{array}$ \\
\hline
\end{tabular}

According to the binary FIGO grading, grade 1 and grade 2 ECs are considered as low-grade and grade 3 ECs are considered as high-grade

\section{Conflict of interest}

All authors declare no conflict of interest.

\section{REFERENCES}

1. WHO, International Agency for Research on Cancer. Corpus uteri. https:// gco.iarc.fr/today/data/factsheets/cancers/24-Corpus-uteri-fact-sheet. pdf (2021-03-01).

2. Siegel R, Miller K, Jemal A. Cancer statistics, 2020. CA Cancer J Clin. 2020; 70(1): 7-30, doi: 10.3322/caac.21590.

3. Colombo N, Creutzberg C, Amant F, et al. ESMO-ESGO-ESTRO Consensus Conference on Endometrial Cancer: diagnosis, treatment and follow-up. Ann Oncol. 2016; 27(1): 16-41, doi: 10.1093/annonc/mdv484, indexed in Pubmed: 26634381.

4. NCCN - Evidence-Based Cancer Guidelines, Oncology Drug Compendium, Oncology Continuing Medical Education. https://www.ncen. org/ (2021-03-01).

5. Kurman RJ, Carcangiu ML, Herrington S. et al.. WHO classification of tumours of female reproductive organs. 4th ed. WHO Press, Lyon 2014.

6. Levine DA. Cancer Genome Atlas Research Network. Integrated genomic characterization of endometrial carcinoma. Nature. 2013; 497(7447): 67-73, doi: 10.1038/nature12113.

7. Talhouk A, McConechy MK, Leung S, et al. A clinically applicable molecular-based classification for endometrial cancers. Br J Cancer. 2015; 113(2): 299-310, doi: 10.1038/bjc.2015.190, indexed in Pubmed: 26172027.

8. Stelloo E, Nout RA, Osse EM, et al. Improved risk assessment by integrating molecular and clinicopathological factors in early-stage endometrial cancer-combined analysis of the PORTEC cohorts. Clin Cancer Res. 2016; 22(16): 4215-4224, doi: 10.1158/1078-0432.CCR-15-2878, indexed in Pubmed: 27006490

9. Talhouk A, McConechy MK, Leung S, et al. Confirmation of ProMisE: a simple, genomics-based clinical classifier for endometrial cancer. Cancer. 2017; 123(5): 802-813, doi: 10.1002/cncr.30496, indexed in Pubmed: 28061006.
10. Church DN, Stelloo E, Nout RA, et al. Prognostic significance of POLE proofreading mutations in endometrial cancer. J Natl Cancer Inst. 2015; 107(1): 402, doi: 10.1093/jnci/dju402, indexed in Pubmed: 25505230.

11. Vermij L, Smit V, Nout $R$, et al. Incorporation of molecular characteristics into endometrial cancer management. Histopathology. 2020; 76(1): 52-63, doi: 10.1111/his.14015, indexed in Pubmed: 31846532.

12. van Gool IC, Eggink FA, Freeman-Mills L, et al. POLE proofreading mutations elicit an antitumor immune response in endometrial cancer. Clin Cancer Res. 2015; 21(14): 3347-3355, doi: 10.1158/1078-0432.CCR-150057, indexed in Pubmed: 25878334.

13. Van Gool IC, Rayner E, Osse EM, et al. Adjuvant treatment for proofreading domain-mutant cancers: sensitivity to radiotherapy, chemotherapy, and nucleoside analogues. Clin Cancer Res. 2018; 24(13): 3197-3203, doi: 10.1158/1078-0432.CCR-18-0266, indexed in Pubmed: 29559562.

14. Creutzberg $C L$, Leon-Castillo A, Boer SMde, et al. Molecular classification of the PORTEC-3 trial for high-risk endometrial cancer: Impact on adjuvant therapy. Ann Oncol. 2019; 30(Suppl 5): v899-v900, doi: 10.1093/annonc/mdz394.060.

15. Kommoss S, McConechy MK, Kommoss F, et al. Final validation of the ProMisE molecular classifier for endometrial carcinoma in a large population-based case series. Ann Oncol. 2018; 29(5): 1180-1188, doi: 10.1093/annonc/mdy058, indexed in Pubmed: 29432521.

16. McMeekin DS, Tritchler DL, Cohn DE, et al. Clinicopathologic significance of mismatch repair defects in endometrial cancer: an NRG oncology/gynecologic oncology group study. J Clin Oncol. 2016; 34(25): 3062-3068, doi: 10.1200/JCO.2016.67.8722, indexed in Pubmed: 27325856.

17. Wortman BG, Creutzberg CL, Putter H, et al. PORTEC Study Group Ten-year results of the PORTEC-2 trial for high-intermediate risk endometrial carcinoma: improving patient selection for adjuvant therapy. Br J Cancer. 2018; 119(9): 1067-1074, doi: 10.1038/s41416-018-0310-8, indexed in Pubmed: 30356126.

18. Kommoss FKf, Karnezis AN, Kommoss F, et al. L1CAM further stratifies endometrial carcinoma patients with no specific molecular risk profile. Br J Cancer. 2018; 119(4): 480-486, doi: 10.1038/s41416-018-0187-6, indexed in Pubmed: 30050154. 
19. Gezer \$ุ, Bayrak BY. Metastatic and synchronous ovarian involvement in low-risk endometrial cancer; clinicopathological analysis with detection of DNA mismatch repair deficiency. Ginekol Pol. 2021 [Epub ahead of print], doi: 10.5603/GP.a2021.0150, indexed in Pubmed: 34541641.

20. Paulino E, de Melo AC. Adjuvant treatment of endometrial cancer in molecular era: Are we ready to move on? Crit Rev Oncol Hematol. 2020; 153: 103016, doi: 10.1016/j.critrevonc.2020.103016, indexed in Pubmed: 32563900.

21. Wortman BG, Creutzberg CL, Putter $\mathrm{H}$, et al. Ten-year results of the PORTEC-2 trial for high-intermediate risk endometrial carcinoma: improving patient selection for adjuvant therapy. Br J Cancer. 2018; 119(9): 1067-1074, doi: 10.1038/s41416-018-0310-8, indexed in Pubmed: 30356126

22. Budak E, Kahraman DS, Budak A, et al. The prognostic significance of serum CA125 levels with ER, PR, P53 and Ki-67 expression in endometrial carcinomas. Ginekol Pol. 2019; 90(12): 675-683, doi: 10.5603/gp.2019.0116.

23. Buza N, English DP, Santin AD, et al. Toward standard HER2 testing of endometrial serous carcinoma: 4-year experience at a large academic center and recommendations for clinical practice. Mod Pathol. 2013;26(12): 16051612, doi: 10.1038/modpathol.2013.113, indexed in Pubmed: 23765245.

24. de Jonge MM, Auguste A, van Wijk LM, et al. Frequent homologous recombination deficiency in high-grade endometrial carcinomas. Clin Cancer Res. 2019; 25(3): 1087-1097, doi: 10.1158/1078-0432.CCR-181443, indexed in Pubmed: 30413523.
25. Howitt BE, Shukla SA, Sholl LM, et al. Association of polymerase e-mutated and microsatellite-instable endometrial cancers with neoantigen load, number of tumor-infiltrating lymphocytes, and expression of PD-1 and PD-L1. JAMA Oncol. 2015; 1(9): 1319-1323, doi: 10.1001/jamaoncol.2015.2151, indexed in Pubmed: 26181000.

26. Azad NS, Gray RJ, Overman MJ, et al. Nivolumab is effective in mismatch repair-deficient noncolorectal cancers: results from arm Z1D-A subprotocol of the NCI-MATCH (EAY131) study. J Clin Oncol. 2020; 38(3): 214-222, doi: 10.1200/JCO.19.00818, indexed in Pubmed: 31765263.

27. Liu JF, Gordon M, Veneris J, et al. Safety, clinical activity and biomarker assessments of atezolizumab from a Phase I study in advanced/recurrent ovarian and uterine cancers. Gynecol Oncol. 2019; 154(2): 314-322, doi: 10.1016/j.ygyno.2019.05.021, indexed in Pubmed: 31204078.

28. Fader $A$, Roque $D$, Siegel $E$, et al. Randomized phase II trial of carboplatin-paclitaxel versus carboplatin-paclitaxel-trastuzumab in uterine serous carcinomas that overexpress human epidermal growth factor receptor 2/neu. J Clin Oncol. 2018; 36(20): 2044-2051, doi: 10.1200/jco.2017.76.5966, indexed in Pubmed: 29584549.

29. Buza N, Roque DM, Santin AD. HER2/neu in endometrial cancer: a promising therapeutic target with diagnostic challenges. Arch Pathol Lab Med. 2014; 138(3): 343-350, doi: 10.5858/arpa.2012-0416-RA, indexed in Pubmed: 24576030.

30. Concin N, Matias-Guiu X, Vergote l, et al. ESGO/ESTRO/ESP guidelines for the management of patients with endometrial carcinoma. Int J Gynecol Cancer. 2021; 31(1): 12-39, doi: 10.1136/ijgc-2020-002230, indexed in Pubmed: 33397713. 